\title{
Characteristics of patients meeting the new definition of pre-capillary pulmonary hypertension (Nice 2018) in a single Japanese pulmonary hypertension center
}

Keiko Yamamoto ${ }^{1,2^{*}}$, Nobuhiro Tanabe ${ }^{1,3}$, Yukiko Takahashi ${ }^{1}$, Akira Naito ${ }^{1}$, Ayumi Sekine ${ }^{1}$, Rika Suda', Takayuki Jujo Sanada ${ }^{1,4}$, Toshihiko Sugiura ${ }^{1}$, Ayako Shigeta ${ }^{1}$, Seiichiro Sakao ${ }^{1}$ and Koichiro Tatsumi ${ }^{1}$

\begin{abstract}
Background: The 6th World Symposium on Pulmonary Hypertension (Nice 2018) proposed a new definition of pre-capillary pulmonary hypertension (PH) as a condition with mean pulmonary artery pressure (mPAP) $>20 \mathrm{mmHg}$, pulmonary artery wedge pressure $\leq 15 \mathrm{mmHg}$, and pulmonary vascular resistance (PVR) $\geq 3$ Wood units (WU). The characteristics and prognosis of patients with pre-capillary $\mathrm{PH}$, according to this new definition, is unclear. Therefore, we determined the characteristics and survival of patients with borderline pre-capillary PH.

Methods: We retrospectively enrolled 683 patients who underwent their first right heart catheterization at Chiba University, Japan. Among them, 489 patients met the pre-capillary PH requirement with mPAP $\geq 25 \mathrm{mmHg}$ (conventional pre-capillary PH group), while 22 patients met the borderline pre-capillary PH criteria (borderline pre-capillary PH group). Additionally, 16 patients with a mean PAP of 20-25 and PVR of 2-3 WU were also examined.

Results: The borderline pre-capillary PH group comprised $4.3 \%$ of the total patients with pre-capillary PH, and the majority was in Group 3 (40.9\%) or 4 (45.5\%). The survival of the borderline pre-capillary PH group tended to be better than that of the conventional pre-capillary PH group. The prognosis of Group3 PH was the worst among the patients with borderline precapillary PH. There was no significant difference in survival between the borderline pre-capillary $\mathrm{PH}$ group with PVR $\geq 3 \mathrm{WU}$ and that with PVR of 2-3 $2 \mathrm{WU}$, although none of the patients in the latter group died due to right heart failure.

Conclusions: This is the first study conducted in a PH center in an Asian country to reveal the characteristics of patients with pre-capillary PH, according to the Nice 2018 definition. They comprised $4.3 \%$ of the total population with pre-capillary PH, and the majority of the pre-capillary PH cases were in either Group3 or 4. The prognosis may be affected by the patients' underlying diseases. Further prospective studies are needed to determine whether the new definition, including the PVR cut-off, is beneficial in clinical practice.
\end{abstract}

Keywords: Pulmonary arterial hypertension, World Symposium on Pulmonary Hypertension (WSPH) 2018, Pulmonary artery wedge pressure, Pulmonary vascular resistance

*Correspondence: shiitake@mx7.ttcn.ne.jp

${ }^{1}$ Department of Respirology, Graduate School of Medicine, Chiba

University, 1-8-1 Inohana Chuou-ku, Chiba 260-8670, Japan

Full list of author information is available at the end of the article

\section{Background}

The World Symposium on Pulmonary Hypertension (WSPH) considered the scientific and clinical knowledge concerning pulmonary hypertension $(\mathrm{PH})$ and proposed 
a new definition for $\mathrm{PH}$ and new treatment strategies. The general purpose of the clinical classification of $\mathrm{PH}$ is to categorize clinical conditions associated with $\mathrm{PH}$ based on similar pathophysiological mechanisms, clinical presentation, hemodynamic characteristics, and therapeutic management. A comprehensive and simplified updated version of the clinical classification of $\mathrm{PH}$ was presented in Nice 2018 [1]. To date, since the first WSPH in 1973, PH has been arbitrarily defined as mean pulmonary arterial pressure $(\mathrm{mPAP}) \geq 25 \mathrm{mmHg}$ at rest. However, recent data obtained for patients undergoing right heart catheterization (RHC) have shown that normal mPAP was $14.0 \pm 3.3 \mathrm{mmHg}$ in healthy subjects, and two standard deviations above this mean value would suggest that mPAP $>20 \mathrm{mmHg}$ is above the upper normal limit $[1,2]$. In addition, the mPAP value is inadequate to define pulmonary vascular disease since this value can be affected by the cardiac output (CO) or pulmonary arterial wedge pressure (PAWP). Considering these aspects, the task force of the 6th WSPH in 2018 proposed that if all three criteria are met, namely, $\mathrm{mPAP}>20 \mathrm{mmHg}$, PAWP $\leq 15 \mathrm{mmHg}$, and pulmonary vascular resistance (PVR) $\geq 3$ Wood units (WU), then the new definition of pre-capillary $\mathrm{PH}$ would be satisfied [1]. A PAWP $>15 \mathrm{mmHg}$ is defined as post-capillary $\mathrm{PH}$, which is considered as isolated $\mathrm{PH}$ when $\mathrm{PVR}<3 \mathrm{WU}$, and combined pre- and post-capillary PH when PVR $\geq 3$ WU. In terms of pulmonary vascular disease, the reliability and validity of this new PH definition have not been defined. Further, the characteristics and survival of patients with pre-capillary $\mathrm{PH}$, diagnosed according to this new definition, are unclear. Moreover, a recent study has revealed that patients with $P V R \geq 2 \mathrm{WU}$ and scleroderma had a significantly poor prognosis [3].Therefore, in our cohort of patients with $\mathrm{PH}$, we determined the characteristics and prognosis of patients with pre-capillary $\mathrm{PH}$ who had pulmonary vascular disease with a modest elevation in mPAP (borderline pre-capillary PH group). This study was conducted in a Japanese $\mathrm{PH}$ center that is associated with respiratory medicine and is one of the high-volume pulmonary endarterectomy (PEA) centers in Japan.

\section{Methods}

\section{Ethics approval and consent to participate}

Patient identity was concealed, and all data were compiled according to the requirements of the Japanese Ministry of Health, Labour and Welfare, which is dedicated to privacy, information technology, and civil rights. Based on the Japanese legislation, the need for informed consent was waived. The study protocol was approved by the Research Ethics Committee of Chiba University School of Medicine (approval number: 2,584). Since 2009, all survivors have provided written informed consent for a prospective cohort study (approval number 826). For patients who died before 2008, written informed consent was not required, in line with the guidelines for retrospective studies in Japan and in accordance with the criteria of the ethics committee of Chiba University Hospital. The study database was anonymized and all experiments were performed in accordance with the relevant guidelines and regulations.

\section{Patients}

Our patients were mainly referred from other hospitals or other departments of Chiba University Hospital. Our PH center is associated with respiratory medicine. Therefore, patients with respiratory diseases suspected of $\mathrm{PH}$ during follow-up were also included. From among them, we conducted a retrospective analysis of the data from patients who had undergone RHC. Indication criteria for RHC were as follows: RHC was conducted when a patient's hypoxia or exercise limitation was considered to have stemmed from $\mathrm{PH}$, and the outcome of RHC seemed to have influenced the patient's treatment, including their eligibility for clinical trials. Among patients with respiratory diseases, RHC was conducted if transplantation was indicated or when RHC would be beneficial. Among the 1542 patients who underwent RHC between 1999 and 2020 at Chiba University, we enrolled 683 patients who were catheterized for the first time at the first diagnosis. The reason for choosing patients from 1999 was because this was the year epoprostenol was approved in Japan; since then, patients have been prescribed selective pulmonary vasodilators. Among these, 531 patients had $\mathrm{mPAP} \geq 25 \mathrm{mmHg}$ (conventional $\mathrm{PH}$ group) and 50 patients had mPAP ranging from 20 to $24 \mathrm{mmHg}$ (borderline $\mathrm{PH}$ group). The non-PH group consisted of 102 patients with mPAP $<20 \mathrm{mmHg}$ (Fig. 1a). We then chose patients with $\mathrm{PH}$ accompanied with pulmonary vascular disease who met the criteria for pre-capillary $\mathrm{PH}(\mathrm{mPAP}>20 \mathrm{mmHg}$ with $\mathrm{PAWP} \leq 15 \mathrm{mmHg}$ and $P V R \geq 3 \mathrm{WU})$. Among the patients with pre-capillary $\mathrm{PH}(\mathrm{n}=511), \mathrm{mPAP} \geq 25 \mathrm{mmHg}$ was observed in 489 (conventional pre-capillary $\mathrm{PH}$ group) and $25>\mathrm{mPAP}>20 \mathrm{mmHg}$ was observed in 22 patients (borderline pre-capillary PH group) (Fig. 1b). We also categorized our cohort into Group 1-5 according to the Nice 2018 classification [1]; the patients were allocated to the groups after evaluation by two pulmonologists. Despite following the new Nice 2018 recommendation, classifying Group 1 and 3 was occasionally difficult, especially when judging morphological or physiological severity.

For Study 1, we compared the characteristics of the conventional $\mathrm{PH}$, borderline $\mathrm{PH}$, and non- $\mathrm{PH}$ groups. The non-PH group was used as a reference since the main aim was to clarify the characteristics of patients 


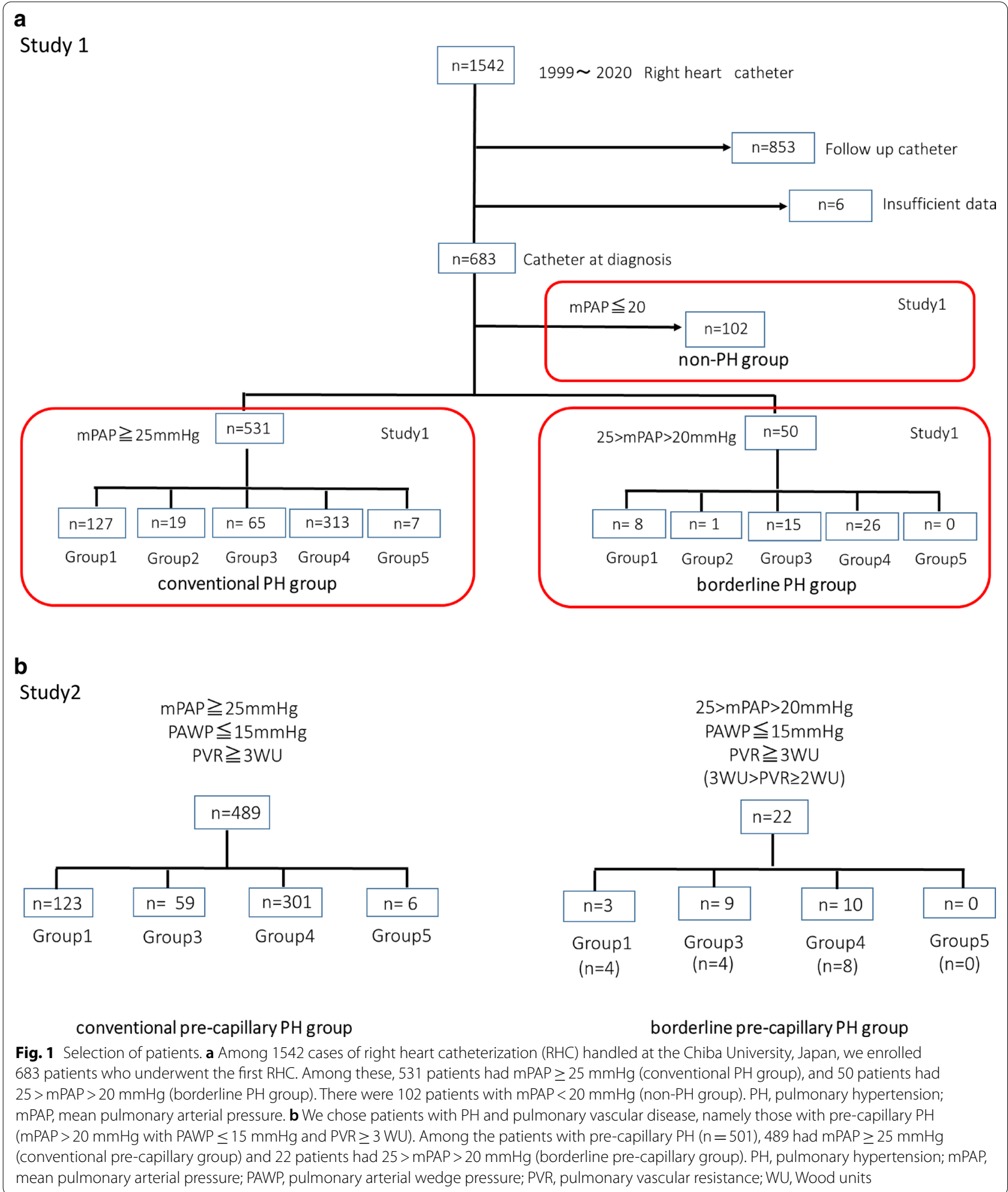

diagnosed with $\mathrm{PH}$ based on the new definition of precapillary PH.

For Study 2, to focus on the pre-capillary nature of $\mathrm{PH}$, the characteristics and survival of the conventional pre-capillary $\mathrm{PH}$ and borderline pre-capillary $\mathrm{PH}$ groups were compared. For Group 3, the PH prognostic factors were also examined. 
Additionally, we compared the survival between the patients with borderline pre-capillary $\mathrm{PH}$ $(25>\mathrm{mPAP}>20 \mathrm{mmHg}$ and $\mathrm{PAWP} \leq 15 \mathrm{mmHg})$ with $\mathrm{PVR} \geq 3 \mathrm{WU}$ and those with $2 \mathrm{WU} \leq \mathrm{PVR}<3 \mathrm{WU}$.

With respect to survival, all-cause mortality or lung transplantation was determined in all participants. We excluded patients with a history of PEA as it has been shown to improve the prognosis of patients with chronic thromboembolic pulmonary hypertension [4].

The follow-up data of RHC in the borderline $\mathrm{PH}$ and borderline pre-capillary $\mathrm{PH}$ groups was also examined.

\section{Statistical analysis}

We used univariate regression analysis to evaluate the baseline characteristics in each group. Student's t-tests and chi-square tests were used to compare continuous variables and categorical variables, respectively. The differences among the three groups were evaluated using one-way analysis of variance (ANOVA). The results are displayed as mean \pm standard deviation or median (interquartile range) for continuous variables, and the number (\%) for categorical variables. The Kaplan-Meier method, log-rank test, and Cox proportional hazards model were used to analyze the mortality and prognostic factors. Multivariate logistic regression analysis was used to identify the factors contributing to poor outcomes. A p-value $<0.05$ was considered statistically significant. All analyses were performed using the JMP Pro software 13.2.0, Japanese version (SAS Institute Inc).

\section{Results}

\section{Conventional $\mathrm{PH}$ and borderline $\mathrm{PH}$ group (Study 1)}

Among the patients who underwent RHC for the first time $(n=683)$, the number of patients in the conventional $\mathrm{PH}$ group was 531 (77.7\%), and that in the borderline $\mathrm{PH}$ group was 50 (8.6\% of total $\mathrm{PH}$ patients) (Fig. 1a).
Most of the patients with borderline $\mathrm{PH}$ were in Group 3 (30\%) and Group 4 (52\%) (Table 1). However, according to the clinical classification, most of the patients with borderline $\mathrm{PH}$ among the total patients in their specific Groups were in Group 3 (15\%), and only $6.8 \%$ were in Group 4 (Table 1).

Detailed analysis, based on etiology, showed that hemodynamics, alveolar-arterial oxygen difference $\left(\mathrm{AaDO}_{2}\right)$, gas exchange impairment, and 6-min walk distance were worst in the conventional group (Table 2). In addition, the partial pressure of arterial carbon dioxide $\left(\mathrm{PaCO}_{2}\right)$ was the lowest in the conventional $\mathrm{PH}$ group. In Group 3, no significant differences in parameters of the ventilatory function were observed among the conventional, borderline $\mathrm{PH}$, and non- $\mathrm{PH}$ groups.

Next, we compared the survival of the conventional $\mathrm{PH}$, borderline, and non-PH groups (Fig. 2). The survival of the conventional $\mathrm{PH}$ group was worse than that of the borderline group, and the worst among all three groups. Analogical tendencies were observed in Groups 1 and 4. However, in Group 3, the 10-year survival of all groups was $<40 \%$. Group 3 showed poor prognosis, even in the non-PH group.

We divided the patients into two sub-groups according to the time of diagnosis (diagnosed in 1999-2009 or 2010-2020), since we recently tended to perform RHC only in patients who may benefit from treatment using vasodilators. The ratio of patients in the non-PH group diagnosed in 1999-2009 was higher than that diagnosed in 2010 (Table 3). Moreover, we analyzed the cause of death among the patients in Group 3. The number of patients who died due to malignant disease or who underwent lung transplantation was higher in the nonPH group than in the PH group (Table 4).

Table 1 Demographic data (Study 1: Conventional PH and borderline PH group, including post-capillary PH)

\begin{tabular}{|c|c|c|c|c|c|c|c|c|c|c|c|c|}
\hline \multirow[t]{2}{*}{ Group } & \multicolumn{3}{|c|}{ Conventional PH } & \multicolumn{3}{|c|}{ Borderline PH } & \multicolumn{3}{|c|}{ Non-PH } & \multicolumn{3}{|c|}{ Total } \\
\hline & $\mathrm{n}$ & $(\%)^{\#}$ & $\%{ }^{\#}$ & $\mathbf{n}$ & $(\%)^{\#}$ & $\%{ }^{\#}$ & $\mathrm{n}$ & $(\%)^{\#}$ & $\%^{\#}$ & $\mathrm{n}$ & $(\%)^{\#}$ & $\% \#$ \\
\hline 1 & 127 & 23.9 & 81.4 & 8 & 16.0 & 5.1 & 21 & 20.6 & 13.5 & 156 & 22.8 & 100 \\
\hline 2 & 19 & 3.6 & 90.5 & 1 & 2.0 & 4.8 & 1 & 1.0 & 4.8 & 21 & 3.1 & 100 \\
\hline 3 & 65 & 12.2 & 65.0 & 15 & 30.0 & 15.0 & 20 & 19.6 & 20.0 & 100 & 14.6 & 100 \\
\hline 4 & 313 & 58.9 & 82.4 & 26 & 52.0 & 6.8 & 41 & 40.2 & 10.8 & 380 & 55.6 & 100 \\
\hline 5 & 7 & 1.3 & 87.5 & 0 & 0.0 & 0.0 & 1 & 1.0 & 12.5 & 8 & 1.2 & 100 \\
\hline Unclassified & 0 & 0.0 & 0.0 & 0 & 0.0 & 0.0 & 18 & 17.6 & 100.0 & 18 & 2.6 & 100 \\
\hline All & 531 & 100.0 & 77.7 & 50 & 100.0 & 7.3 & 102 & 100.0 & 14.9 & 683 & 100.0 & 100 \\
\hline
\end{tabular}

PH pulmonary hypertension

\# (\%) each clinical classification group in all groups; * \% of conventional PH group, borderline PH group, non-PH group among each clinical classification group 
Table 2 Baseline characteristics (Study 1; Conventional PH and borderline PH group, including post-capillary PH)

\begin{tabular}{|c|c|c|c|c|c|c|c|c|c|c|c|c|c|}
\hline \multirow[t]{2}{*}{ Total } & \multirow[b]{2}{*}{$\mathbf{n}$} & \multicolumn{3}{|l|}{ Conventional } & \multirow[b]{2}{*}{$\mathrm{n}$} & \multicolumn{3}{|l|}{ Borderline } & \multirow[b]{2}{*}{$\mathrm{n}$} & \multicolumn{2}{|l|}{ Non-PH } & & \multirow[t]{2}{*}{$p$-value } \\
\hline & & & & & & & & & & & & & \\
\hline Age & 531 & 56.7 & \pm & 15.2 & 50 & 56.2 & \pm & 15.8 & 102 & 57.8 & \pm & 16.1 & 0.7591 \\
\hline $\operatorname{Sex}(F / M)$ & 531 & 373 & / & 158 & 50 & 29 & / & 21 & 102 & 70 & / & 32 & 0.2152 \\
\hline $\mathrm{mPAP}(\mathrm{mmHg})$ & 531 & 42.9 & \pm & 11.8 & 50 & 22.6 & \pm & 1.2 & 102 & 16.2 & \pm & 3.2 & $<0.0001$ \\
\hline PVR (WU) & 531 & 8.7 & \pm & 4.7 & 50 & 3.0 & \pm & 1.3 & 102 & 2.2 & \pm & 0.9 & $<0.0001$ \\
\hline $\mathrm{PAWP}(\mathrm{mmHg})$ & 531 & 8.1 & \pm & 3.8 & 50 & 8.0 & \pm & 3.9 & 102 & 5.9 & \pm & 3.0 & $<0.0001$ \\
\hline $\mathrm{CO}(\mathrm{L} / \mathrm{min})$ & 531 & 4.5 & \pm & 1.5 & 50 & 5.4 & \pm & 1.8 & 102 & 5.1 & \pm & 1.4 & $<0.0001$ \\
\hline $6 \mathrm{MWD}(\mathrm{m})$ & 413 & 362.0 & \pm & 105.3 & 32 & 410.4 & \pm & 105.5 & 51 & 429.0 & \pm & 112.6 & $<0.0001$ \\
\hline$\% \mathrm{VC}$ & 482 & 85.4 & \pm & 21.5 & 39 & 88.6 & \pm & 26.0 & 86 & 88.9 & \pm & 24.3 & 0.3056 \\
\hline FEV1.0\% & 182 & 75.5 & \pm & 11.4 & 39 & 77.6 & \pm & 18.5 & 86 & 78.0 & \pm & 15.0 & 0.176 \\
\hline$\% \mathrm{DLCO} / \mathrm{NA}$ & 455 & 76.5 & \pm & 27.4 & 38 & 74.1 & \pm & 25.2 & 79 & 85.1 & \pm & 31.1 & 0.0297 \\
\hline $\mathrm{PaO}_{2}(\mathrm{mmHg})$ & 517 & 65.3 & \pm & 22.1 & 50 & 74.3 & \pm & 13.0 & 99 & 82.5 & \pm & 17.6 & $<0.0001$ \\
\hline $\mathrm{PaCO}_{2}(\mathrm{mmHg})$ & 517 & 38.5 & \pm & 6.4 & 50 & 40.6 & \pm & 7.2 & 99 & 40.2 & \pm & 5.3 & 0.0051 \\
\hline $\mathrm{P}_{\mathrm{V}} \mathrm{O}_{2}(\mathrm{mmHg})$ & 515 & 34.7 & \pm & 4.9 & 50 & 38.1 & \pm & 4.8 & 99 & 40.2 & \pm & 7.9 & $<0.0001$ \\
\hline $\mathrm{O}_{2}$ administration $(+)$ & & $75(14.1 \%)$ & & & & $3(6.0 \%)$ & & & & $8(7.8 \%)$ & & & 0.0536 \\
\hline $\mathrm{AaDo}_{2}(\mathrm{mmHg})$ & 516 & 39.0 & \pm & 25.0 & 50 & 17.6 & \pm & 11.1 & 99 & 19.6 & \pm & 16.5 & $<0.0001$ \\
\hline $\begin{array}{l}\text { WHO-FC } \\
(\mathrm{I} / \mathrm{II} / \mathrm{II} / \mathrm{IV})\end{array}$ & & $(6 / 259 / 255 / 11)$ & & & & $(0 / 37 / 13 / 0)$ & & & & $(16 / 62 / 23 / 1)$ & & & $<0.0001$ \\
\hline Vasodilators $( \pm)$ & 531 & 316 & / & 215 & 50 & 8 & / & 42 & 102 & 2 & / & 100 & $<0.0001$ \\
\hline \multicolumn{14}{|l|}{ Group 1} \\
\hline Age & 127 & 48.6 & \pm & 18.1 & 8 & 49.5 & \pm & 6.2 & 21 & 58.7 & \pm & 3.9 & 0.0546 \\
\hline $\operatorname{Sex}(F / M)$ & 127 & 103 & / & 24 & 8 & 5 & / & 3 & 21 & 20 & / & 1 & 0.0346 \\
\hline $\mathrm{mPAP}(\mathrm{mmHg})$ & 127 & 44.3 & \pm & 12.1 & 8 & 22.8 & \pm & 1.3 & 21 & 16.6 & \pm & 2.8 & $<0.0001$ \\
\hline PVR (WU) & 127 & 8.7 & \pm & 4.8 & 8 & 3.0 & \pm & 1.3 & 21 & 2.2 & \pm & 1.1 & $<0.0001$ \\
\hline $\mathrm{PAWP}(\mathrm{mmHg})$ & 127 & 7.5 & \pm & 4.5 & 8 & 7.5 & \pm & 4.5 & 21 & 5.8 & \pm & 3.1 & 0.0337 \\
\hline $\mathrm{CO}(\mathrm{L} / \mathrm{min})$ & 127 & 4.8 & \pm & 1.7 & 8 & 6.1 & \pm & 3.1 & 21 & 5.3 & \pm & 1.5 & 0.1076 \\
\hline $6 \mathrm{MWD}(\mathrm{m})$ & 97 & 398.5 & \pm & 108.6 & 6 & 434.2 & \pm & 61.6 & 14 & 455.2 & \pm & 111.2 & 0.152 \\
\hline VC,\% predicted & 115 & 85.2 & \pm & 16.9 & 6 & 89.8 & \pm & 9.8 & 19 & 86.1 & \pm & 14.9 & 0.7914 \\
\hline FEV1.0,\% predicted & 115 & 78.9 & \pm & 9.8 & 6 & 79.2 & \pm & 8.9 & 19 & 82.1 & \pm & 10.1 & 0.432 \\
\hline DLCO/NA,\% predicted & 111 & 74.7 & \pm & 25.7 & 6 & 76.3 & \pm & 16.5 & 18 & 81.2 & \pm & 39.3 & 0.659 \\
\hline $\mathrm{PaO}_{2}(\mathrm{mmHg})$ & 125 & 72.6 & \pm & 18.9 & 8 & 75.9 & \pm & 13.8 & 21 & 87.7 & \pm & 15.8 & 0.0018 \\
\hline $\mathrm{PaCO}_{2}(\mathrm{mmHg})$ & 125 & 36.6 & \pm & 5.3 & 8 & 39.6 & \pm & 1.2 & 21 & 40.7 & \pm & 6.6 & 0.0037 \\
\hline $\mathrm{P}_{\mathrm{V}} \mathrm{O}_{2}(\mathrm{mmHg})$ & 123 & 37.7 & \pm & 5.1 & 8 & 41.8 & \pm & 8.2 & 21 & 42.3 & \pm & 5.5 & 0.0005 \\
\hline $\mathrm{O}_{2}$ administration $(+)$ & & $18(14.2 \%)$ & & & & $0(0.0 \%)$ & & & & $0(0.0 \%)$ & & & 0.019 \\
\hline $\mathrm{AaDo}_{2}(\mathrm{mmHg})$ & 125 & 33.8 & \pm & 19.3 & 8 & 27.1 & \pm & 13.8 & 21 & 14.0 & \pm & 12.4 & $<0.0001$ \\
\hline $\begin{array}{l}\text { WHO-FC } \\
(\mathrm{I} / \mathrm{II} / \mathrm{II} / \mathrm{IV})\end{array}$ & & $(3 / 83 / 40 / 1)$ & & & & $(0 / 7 / 1 / 0)$ & & & & $(1 / 18 / 2 / 0)$ & & & 0.2859 \\
\hline Vasodilators $( \pm)$ & 127 & 95 & 94 & 33 & 8 & 4 & / & 4 & 21 & 1 & / & 20 & $<0.0001$ \\
\hline Underlying diseases & & & (\%) & & & & $(\%)$ & & & & (\%) & & \\
\hline IPAH/HPAH/PVOD/PCH & & 57 & 44.9 & & & - & - & & & - & - & & \\
\hline CTD & & 39 & 30.7 & & & 4 & 50.0 & & & 8 & 38.1 & & \\
\hline Congenital & & 19 & 15.0 & & & 2 & 25.0 & & & 3 & 14.3 & & \\
\hline Portal hypertension & & 12 & 9.4 & & & 1 & 12.5 & & & 1 & 4.8 & & \\
\hline drug/HIV & & 2 & 1.6 & & & 0 & 0.0 & & & 0 & 0.0 & & \\
\hline unknown & & - & - & & & 1 & 12.5 & & & 9 & 42.9 & & \\
\hline \multicolumn{14}{|l|}{ Group 3} \\
\hline Age & 65 & 61.5 & \pm & 13.4 & 15 & 59.7 & \pm & 18.7 & 20 & 60.1 & \pm & 13.8 & 0.8723 \\
\hline $\operatorname{Sex}(F / M)$ & 65 & 33 & / & 32 & 15 & 6 & / & 9 & 20 & 5 & / & 15 & 0.0321 \\
\hline $\mathrm{mPAP}(\mathrm{mmHg})$ & 65 & 35.7 & \pm & 10.8 & 15 & 22.4 & \pm & 1.4 & 20 & 16.0 & \pm & 3.4 & $<0.0001$ \\
\hline PVR (WU) & 65 & 7.0 & \pm & 5.2 & 15 & 3.3 & \pm & 1.2 & 20 & 2.5 & \pm & 0.9 & $<0.0001$ \\
\hline
\end{tabular}


Table 2 (continued)

\begin{tabular}{|c|c|c|c|c|c|c|c|c|c|c|c|c|c|}
\hline \multirow[t]{2}{*}{ Total } & \multirow[b]{2}{*}{$\mathbf{n}$} & \multicolumn{3}{|l|}{ Conventional } & \multirow[b]{2}{*}{$\mathrm{n}$} & \multicolumn{3}{|l|}{ Borderline } & \multirow[b]{2}{*}{$\mathbf{n}$} & \multicolumn{2}{|l|}{ Non-PH } & & \multirow[t]{2}{*}{$p$-value } \\
\hline & & & & & & & & & & & & & \\
\hline PAWP $(m m H g)$ & 65 & 7.1 & \pm & 3.7 & 15 & 7.1 & \pm & 3.7 & 20 & 4.8 & \pm & 2.6 & 0.0016 \\
\hline $\mathrm{CO}(\mathrm{L} / \mathrm{min})$ & 65 & 4.7 & \pm & 1.6 & 15 & 5.2 & \pm & 1.8 & 20 & 47.0 & \pm & 1.2 & 0.5076 \\
\hline $6 \mathrm{MWD}(\mathrm{m})$ & 38 & 281.5 & \pm & 87.2 & 10 & 310.2 & \pm & 121.7 & 14 & 376.1 & \pm & 99.4 & 0.01 \\
\hline VC,\% predicted & 58 & 58.7 & \pm & 24.0 & 13 & 68.3 & \pm & 28.6 & 20 & 71.5 & \pm & 26.6 & 0.1134 \\
\hline FEV1.0,\% predicted & 58 & 73.2 & \pm & 18.9 & 13 & 75.4 & \pm & 30.9 & 20 & 69.9 & \pm & 24.2 & 0.7678 \\
\hline DLCO/NA,\% predicted & 47 & 43.3 & \pm & 29.1 & 12 & 53.3 & \pm & 27.4 & 17 & 69.3 & \pm & 22.4 & 0.0052 \\
\hline $\mathrm{PaO}_{2}(\mathrm{mmHg})$ & 59 & 66.1 & \pm & 38.0 & 15 & 67.0 & \pm & 14.6 & 19 & 81.3 & \pm & 18.4 & 0.1991 \\
\hline $\mathrm{PaCO}_{2}(\mathrm{mmHg})$ & 59 & 46.8 & \pm & 10.5 & 15 & 45.6 & \pm & 9.9 & 19 & 42.2 & \pm & 5.8 & 0.1983 \\
\hline $\mathrm{P}_{\mathrm{V}} \mathrm{O}_{2}(\mathrm{mmHg})$ & 59 & 35.3 & \pm & 4.4 & 15 & 35.5 & \pm & 2.9 & 19 & 38.8 & \pm & 5.4 & 0.0134 \\
\hline $\mathrm{O}_{2}$ administration $(+)$ & 65 & $1(6.7 \%)$ & & & & 18(27.7\%) & & & & $3(15.0 \%)$ & & & 0.1096 \\
\hline $\mathrm{AaDo}_{2}(\mathrm{mmHg})$ & 58 & 28.9 & \pm & 47.3 & 15 & 29.0 & \pm & 13.3 & 19 & 18.7 & \pm & 17.7 & 0.5993 \\
\hline $\begin{array}{l}\text { WHO-FC } \\
(1 / I / I / I I / / V)\end{array}$ & & $(0 / 14 / 49 / 2)$ & & & & $(0 / 6 / 9 / 0)$ & & & & $(1 / 4 / 14 / 1)$ & & & 0.3784 \\
\hline Vasodilators & 65 & 35 & / & 30 & 15 & 0 & / & 15 & 20 & 0 & / & 20 & $<0.0001$ \\
\hline Underlying diseases & & & $(\%)$ & & & & (\%) & & & & $(\%)$ & & \\
\hline IP & & 38 & 58.5 & & & 7 & 46.7 & & & 8 & 40.0 & & \\
\hline COPD & & 13 & 20.0 & & & 5 & 33.3 & & & 7 & 35.0 & & \\
\hline $\mathrm{BE}$ & & 8 & 12.3 & & & 1 & 6.7 & & & 0 & 0.0 & & \\
\hline Others & & 6 & 9.2 & & & 2 & 13.3 & & & 1 & 5.0 & & \\
\hline \multicolumn{14}{|l|}{ Group 4} \\
\hline Age & 313 & 58.3 & \pm & 13.1 & 26 & 55.5 & \pm & 14.4 & 41 & 57.0 & \pm & 17.4 & 0.5267 \\
\hline $\operatorname{Sex}(F / M)$ & 313 & 223 & / & 90 & 26 & 18 & / & 8 & 41 & 29 & / & 12 & 0.9757 \\
\hline mPAP $(\mathrm{mmHg})$ & 313 & 44.3 & \pm & 11.4 & 26 & 22.7 & \pm & 1.2 & 41 & 16.5 & \pm & 3.0 & $<0.0001$ \\
\hline PVR (WU) & 313 & 9.4 & \pm & 4.5 & 26 & 3.0 & \pm & 1.3 & 41 & 2.2 & \pm & 0.8 & $<0.0001$ \\
\hline PAWP (mmHg) & 313 & 7.6 & \pm & 3.2 & 26 & 8.3 & \pm & 3.5 & 41 & 5.9 & \pm & 2.7 & 0.0018 \\
\hline $\mathrm{CO}(\mathrm{L} / \mathrm{min})$ & 313 & 4.3 & \pm & 1.1 & 26 & 5.2 & \pm & 1.3 & 41 & 5.0 & \pm & 1.1 & $<0.0001$ \\
\hline $6 \mathrm{MWD}(\mathrm{m})$ & 264 & 359.4 & \pm & 100.2 & 15 & 465.7 & \pm & 54.8 & 15 & 451.5 & \pm & 119.5 & $<0.0002$ \\
\hline VC,\% predicted & 290 & 91.8 & \pm & 18.0 & 19 & 103.4 & \pm & 17.1 & 31 & 101.3 & \pm & 21.1 & 0.0012 \\
\hline FEV1.0,\% predicted & 290 & 75.0 & \pm & 9.4 & 19 & 79.0 & \pm & 7.8 & 31 & 78.2 & \pm & 9.1 & 0.0631 \\
\hline DLCO/NA,\% predicted & 278 & 82.5 & \pm & 23.8 & 19 & 85.2 & \pm & 18.0 & 30 & 96.9 & \pm & 19.2 & 0.0054 \\
\hline $\mathrm{PaO}_{2}(\mathrm{mmHg})$ & 308 & 60.9 & \pm & 18.0 & 26 & 78.2 & \pm & 10.3 & 40 & 78.5 & \pm & 15.8 & $<0.0001$ \\
\hline $\mathrm{PaCO}_{2}(\mathrm{mmHg})$ & 308 & 37.6 & \pm & 4.4 & 26 & 38.5 & \pm & 4.4 & 40 & 39.7 & \pm & 4.7 & 0.0114 \\
\hline $\mathrm{P}_{\mathrm{V}} \mathrm{O}_{2}(\mathrm{mmHg})$ & 308 & 33.3 & \pm & 4.3 & 26 & 38.7 & \pm & 3.4 & 40 & 39.2 & \pm & 3.4 & $<0.0001$ \\
\hline $\mathrm{O}_{2}$ administration(+) & & $36(9.5 \%)$ & & & & $2(7.7 \%)$ & & & & $0(0.0 \%)$ & & & 0.0115 \\
\hline $\mathrm{AaDo}_{2}(\mathrm{mmHg})$ & 308 & 44.4 & \pm & 18.9 & 26 & 26.1 & \pm & 8.4 & 40 & 24.3 & \pm & 14.8 & $<0.0001$ \\
\hline $\begin{array}{l}\text { WHO-FC } \\
(\mathrm{I} / \mathrm{II} / \mathrm{III} / \mathrm{IV})\end{array}$ & & $(3 / 147 / 156 / 7)$ & & & & $(0 / 24 / 2 / 0)$ & & & & $(8 / 30 / 3 / 0)$ & & & $<0.0001$ \\
\hline Vasodilators & 313 & 177 & / & 136 & 26 & 4 & / & 22 & 41 & 1 & / & 40 & $<0.0001$ \\
\hline PEA & 313 & 158 & / & 155 & 26 & 2 & / & 24 & 41 & 1 & / & 40 & $<0.0001$ \\
\hline BPA & 313 & 53 & / & 260 & 26 & 0 & / & 26 & 41 & 0 & / & 41 & $<0.0001$ \\
\hline Underlying diseases & & & $(\%)$ & & & & (\%) & & & & $(\%)$ & & \\
\hline$P E$ & & 289 & 92.3 & & & 22 & 84.6 & & & 35 & 85.4 & & \\
\hline Pulmonary stenosis & & 22 & 7.0 & & & 4 & 15.4 & & & 5 & 12.2 & & \\
\hline Others & & 2 & 0.6 & & & 0 & 0.0 & & & 1 & 2.4 & & \\
\hline
\end{tabular}

mPAP, mean pulmonaryarterial pressure; PVR, pulmonary vascular resistance; PAWP, pulmonary arterial wedge pressure; $C O$, cardiac output; 6MWD, 6-min walk distance; \%VC, percent vital capacity; FEV1.0\%, percent predicted forced expiratory volume in one second; \%DLCO/VA, diffusing capacity of carbon monoxide by the alveolar volume; $\mathrm{PaO}_{2}$, partial pressure of arterial oxygen; $\mathrm{PaCO}_{2}$, partial pressure of arterial carbon dioxide; $\mathrm{PvO}_{2}$, mixed venous oxygen tension; AaDO arterial oxygen difference; WHO-FC, World Health Organization Functional Class; IPAH, idiopathic pulmonary hypertension; HPAH, hereditary pulmonary hypertension; PVOD, pulmonary veno-occlusive disease; $\mathrm{PCH}$, pulmonary capillary hemangiomatosis; CTD, connective tissue disease; ILD, interstitial lung disease; COPD, chronic obstructive pulmonary disease; $\mathrm{BE}$, bronchiectasis; $\mathrm{PEA}$, pulmonary endarterectomy; $\mathrm{PE}$, pulmonary embolism 
Fig. 2 Survival (Study 1: Conventional PH and borderline PH group including post-capillary PH). The survival of the conventional PH group was the worst among the 3 groups $(p=0.0085)$. There was no significant difference in the survival between the borderline group and the non-PH group. $\mathrm{PH}$, pulmonary hypertension

\section{Characteristics and survival of pre-capillary $\mathrm{PH}$ patients (Study 2)}

The number of patients in the conventional pre-capillary PH group was 489 (71.6\%). Twenty-two patients (3.2\% of the total patients, including the patients with non-PH; $4.3 \%$ of the patients with total pre-capillary $\mathrm{PH}$ ) were included in the borderline pre-capillary $\mathrm{PH}$ group (Fig. 1b and Table 5). Similar to Study 1, most of the patients with borderline pre-capillary $\mathrm{PH}$ belonged to Groups 3 (40.9\%) and 4 (45.5\%). However, among the total patients with pre-capillary $\mathrm{PH}$, most of the patients with borderline pre-capillary PH belonged to Group 3 (13.2\%), and only $3.2 \%$ patients belonged to Group 4 (Table 5 ).

Regarding baseline characteristics, in addition to hemodynamics, partial pressure of arterial oxygen $\left(\mathrm{PaO}_{2}\right)$, partial pressure of mixed venous oxygen $\left(\mathrm{PvO}_{2}\right)$, and $\mathrm{AaDO}_{2}$ in the conventional pre-capillary $\mathrm{PH}$ group were significantly worse than those in the borderline precapillary $\mathrm{PH}$ group. In addition, $\mathrm{PaCO}_{2}$ was significantly lower in the conventional pre-capillary $\mathrm{PH}$ group. There was no significant difference in the ventilatory function between the two groups.

Focusing on each etiological group, the conventional pre-capillary PH group showed more severe hemodynamics and gas exchange impairment than the borderline pre-capillary PH group in Groups 1, 3, and 4. A significantly lower $\mathrm{PaCO}_{2}$ in the conventional pre-capillary $\mathrm{PH}$ group was observed only in Group 1 relative to that in the other Groups. Even in Group 3, there was no significant difference in the ventilatory function parameters between the two groups (Table 6).

Furthermore, the survival of the conventional precapillary $\mathrm{PH}$ group was worse than that of the borderline pre-capillary PH group; however, it did not reach statistical significance. The same tendency was observed in Group 1, 3, and 4 (Fig. 3). The 10-year-survival was the worst in Group 3 in the conventional PH group (Group 1, 50.1\%; Group 3, 0.00\%; Group 4, 68.0\%) and the borderline pre-capillary PH group (Group 1, 50.9\%; Group 3, 0.0\%; Group 4, 67.0\%). Age and conventional PH vs. borderline $\mathrm{PH}$ were poor prognostic factors; however, no parameter was statistically significant (Table 7).

There was no significant difference in survival between the patients with borderline pre-capillary $\mathrm{PH}$

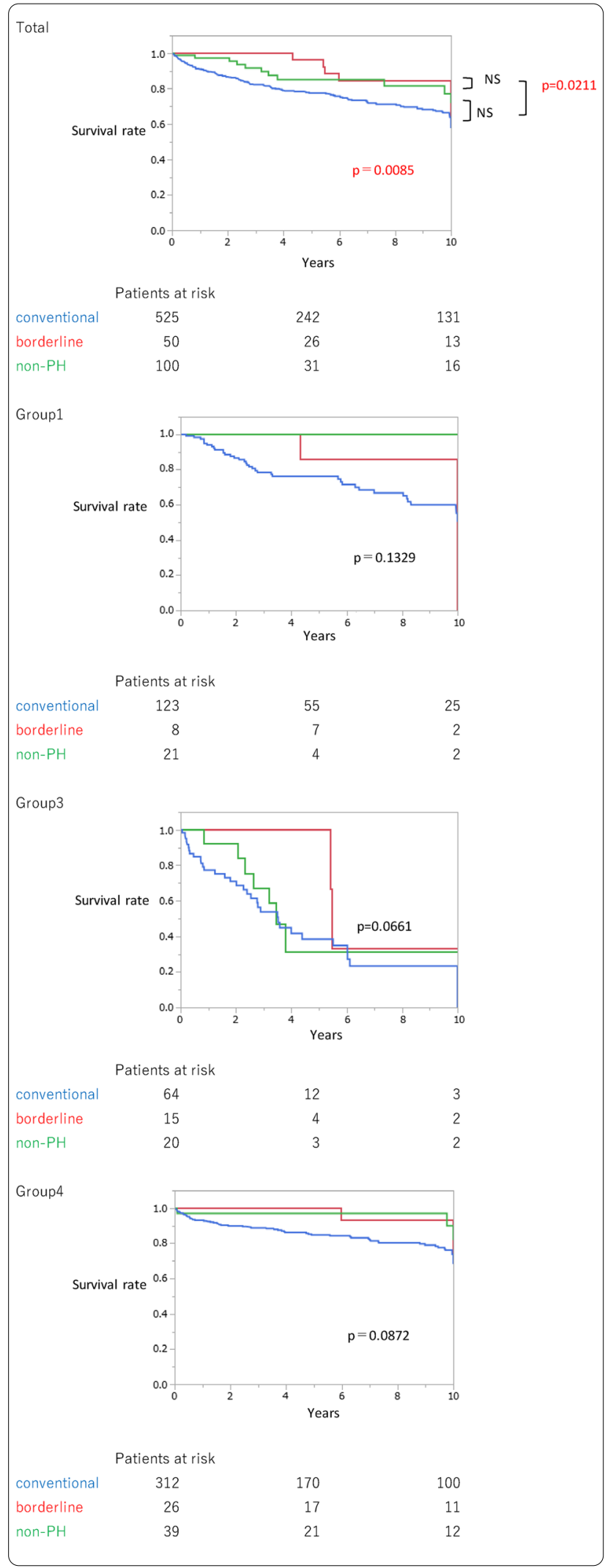


Table 3 Time of diagnosis in Group 3

\begin{tabular}{llllll}
\hline & $\sim 2009$ & & & $\sim 2010$ & \\
\cline { 2 - 3 } \cline { 5 - 6 } & $\mathbf{n}$ & \% & & $\mathbf{n}$ & \% \\
\hline Conventional & 9 & 40.9 & 56 & 71.8 \\
Borderline & 4 & 18.2 & 11 & 14.1 \\
Non-PH & 9 & 40.9 & 11 & 14.1 \\
Total & 22 & & 78 & \\
\hline
\end{tabular}

$p=0.0168$

$(25>\mathrm{mPAP}>20 \mathrm{mmHg}$ and PAWP $\leq 15 \mathrm{mmHg})$ with $\mathrm{PVR} \geq 3$ WU $(\mathrm{n}=22)$ and those with $2 \leq \mathrm{PVR}<3 \mathrm{WU}$ $(\mathrm{n}=16)$ (Fig. 4). Among them, five patients died; however, none of them succumbed to right heart failure (PVR $\geq 3$ : 1 , pneumonia; $2 \leq \mathrm{PVR}<3 \mathrm{WU}: 4$, malignancy).

\section{Follow-up RHC data of patients with borderline $\mathrm{PH}$} and those with borderline pre-capillary $\mathrm{PH}$

Among the patients with borderline $\mathrm{PH}$, follow-up RHC was performed when the clinical condition was considered to worsen. Among 50 patients with borderline $\mathrm{PH}$ and 22 with borderline pre-capillary $\mathrm{PH}$, only five patients underwent follow-up RHC (borderline $\mathrm{PH}=4$; borderline pre-capillary $\mathrm{PH}=1$ ), and only two patients were prescribed pulmonary vasodilators (borderline $\mathrm{PH}=1$; borderline pre-capillary $\mathrm{PH}=1$ ).
Only one patient's condition improved after treatment (Table 8).

\section{Discussion}

This is the first study conducted in a $\mathrm{PH}$ center in an Asian country to reveal the characteristics and survival of patients diagnosed with pre-capillary $\mathrm{PH}$, according to the new diagnostic criteria proposed at the WSPH 2018. The borderline pre-capillary $\mathrm{PH}$ group accounted for $3.2 \%$ (22 in 683) of the total patient population who underwent their first $\mathrm{RHC}$, and $4.3 \%$ of all patients with pre-capillary $\mathrm{PH}$ (22 in 511). The borderline $\mathrm{PH}$ group comprised 8.6\% (50 in 581) of all patients with PH. Most of the patients in the borderline pre-capillary $\mathrm{PH}$ group belonged to Group 3 and 4. The survival of the borderline pre-capillary $\mathrm{PH}$ group tended to be better than that of the conventional pre-capillary $\mathrm{PH}$ group. Further, the prognosis of $\mathrm{PH}$ in Group 3 was the worst among the patients with borderline precapillary $\mathrm{PH}$.

Most of the previous studies have focused on patients with borderline $\mathrm{PH}(25>\mathrm{mPAP}>20 \mathrm{mmHg})$, and not on the pre-capillary nature of $\mathrm{PH}$. In previous studies, the percentage of borderline $\mathrm{PH}$ has ranged from 4.2 to $18 \%$ among all patients and $4.5-22.6 \%$ of all patients with mPAP $>20 \mathrm{mmHg}$ [5-7]. Further, Group $2 \mathrm{PH}$ corresponds to the most common form of conventional $\mathrm{PH}$ due to left heart failure [8]. Previous studies have shown

Table 4 Causes of death or lung transplantation in Group 3

\begin{tabular}{|c|c|c|c|c|c|c|c|c|c|c|c|}
\hline & \multicolumn{2}{|c|}{ RHF } & \multicolumn{2}{|c|}{ Lung disease } & \multicolumn{2}{|c|}{ Malignancy } & \multicolumn{2}{|c|}{$\begin{array}{l}\text { Lung } \\
\text { transplantation }\end{array}$} & \multicolumn{2}{|c|}{ Others } & \multirow[t]{2}{*}{ Total } \\
\hline & $\mathrm{n}$ & $\%$ & $\mathrm{n}$ & $\%$ & $\mathrm{n}$ & $\%$ & $\mathbf{n}$ & $\%$ & $\mathrm{n}$ & $\%$ & \\
\hline Conventional & 10 & 29.4 & 14 & 41.2 & 1 & 2.9 & 2 & 5.9 & 7 & 20.6 & 34 \\
\hline Borderline & 0 & 0.0 & 1 & 50.0 & 0 & 0.0 & 0 & 0.0 & 1 & 50.0 & 2 \\
\hline Non-PH & 0 & 0.0 & 3 & 42.9 & 1 & 14.3 & 2 & 28.6 & 1 & 14.3 & 7 \\
\hline
\end{tabular}

$\mathrm{PH}$, pulmonary hypertension; RHF, right heart failure

Table 5 Demographic data (Study 2: Conventional PH and borderline PH group in pre-capillary PH)

\begin{tabular}{|c|c|c|c|c|c|c|c|c|c|}
\hline \multirow[b]{2}{*}{ Group } & \multicolumn{3}{|c|}{ Conventional pre-capillary PH } & \multicolumn{3}{|c|}{ Borderline pre-capillary PH } & \multicolumn{3}{|c|}{ Total pre-capillary PH } \\
\hline & $\mathbf{n}$ & $(\%)^{\#}$ & $\%^{\#}$ & $\mathbf{n}$ & $(\%)^{\#}$ & $\%^{\#}$ & $\mathbf{n}$ & $(\%)^{\#}$ & $\%^{\#}$ \\
\hline 1 & 123 & 25.2 & 97.6 & 3 & 2.4 & 13.6 & 126 & 24.7 & 100 \\
\hline 3 & 59 & 12.1 & 86.8 & 9 & 13.2 & 40.9 & 68 & 13.3 & 100 \\
\hline 4 & 301 & 61.6 & 96.8 & 10 & 3.2 & 45.5 & 311 & 60.9 & 100 \\
\hline 5 & 6 & 1.2 & 100.0 & 0 & 0.0 & 0.0 & 6 & 1.2 & 100 \\
\hline Total & 489 & 100.0 & 95.7 & 22 & 4.3 & 100.0 & 511 & 100.0 & 100 \\
\hline
\end{tabular}

$\mathrm{PH}$, pulmonary hypertension

\# (\%): each clinical classification group in all groups; *\%: conventional pre-capillary PH group and borderline pre-capillary PH group among each clinical classification group 
Table 6 Baseline characteristics (Study 2: Conventional PH and borderline PH group in pre-capillary PH)

\begin{tabular}{|c|c|c|c|c|c|c|c|c|c|}
\hline Total & $\mathbf{N}$ & Conventional $\mathrm{p}$ & capillry PH & & $\mathrm{n}$ & Borderline & e-capillary PH & & p-value \\
\hline Age & 489 & 56.1 & \pm & 15.2 & 22 & 58.2 & \pm & 13.9 & 0.5342 \\
\hline $\operatorname{Sex}(F / M)$ & 489 & 347 & / & 142 & 22 & 15 & / & 7 & 0.7808 \\
\hline $\mathrm{mPAP}(\mathrm{mmHg})$ & 489 & 43.7 & \pm & 11.7 & 22 & 23.0 & \pm & 1.2 & $<0.0001$ \\
\hline PVR (WU) & 489 & 9.2 & \pm & 4.6 & 22 & 4.2 & \pm & 0.9 & $<0.0001$ \\
\hline PAWP $(m m H g)$ & 489 & 7.5 & \pm & 3.1 & 22 & 5.7 & \pm & 2.9 & 0.0057 \\
\hline $\mathrm{CO}(\mathrm{L} / \mathrm{min})$ & 489 & 4.3 & \pm & 1.2 & 22 & 4.2 & \pm & 0.9 & 0.5689 \\
\hline $6 \mathrm{MWD}(\mathrm{m})$ & 449 & 360.1 & \pm & 104.7 & 12 & 403.2 & \pm & 11.3 & 0.1704 \\
\hline VC,\% predicted & 444 & 86.7 & \pm & 21.2 & 17 & 86.0 & \pm & 28.6 & 0.8827 \\
\hline FEV1.0,\% predicted & 444 & 75.8 & \pm & 10.8 & 17 & 73.9 & \pm & 17.3 & 0.4922 \\
\hline DLCO/NA,\% predicted & 420 & 75.8 & \pm & 27.3 & 16 & 70.7 & \pm & 21.8 & 0.4577 \\
\hline $\mathrm{PaO}_{2}(\mathrm{mmHg})$ & 476 & 64.4 & \pm & 21.5 & 22 & 74.6 & \pm & 14.2 & 0.0281 \\
\hline $\mathrm{PaCO}_{2}(\mathrm{mmHg})$ & 476 & 38.2 & \pm & 6.1 & 22 & 41.4 & \pm & 9.3 & 0.0212 \\
\hline $\mathrm{P}_{\mathrm{V}} \mathrm{O}_{2}(\mathrm{mmHg})$ & 475 & 34.4 & \pm & 4.7 & 22 & 37.1 & \pm & 3.7 & 0.0087 \\
\hline $\mathrm{O}_{2}$ administration $(+)$ & & & $71(14.5 \%)$ & & & & $1(4.6 \%)$ & & 0.1348 \\
\hline $\mathrm{AaDo}_{2}(\mathrm{mmHg})$ & 475 & 40.3 & \pm & 23.8 & 22 & 26.3 & \pm & 10.3 & 0.0063 \\
\hline $\begin{array}{l}\text { WHO-FC } \\
(\mathrm{I} / \mathrm{II} / \mathrm{II} / \mathrm{IV})\end{array}$ & & $(6 / 231 / 241 / 11)$ & & & & $(0 / 17 / 5 / 0)$ & & & 0.0344 \\
\hline Vasodilators $( \pm)$ & 489 & 301 & / & 188 & 22 & 5 & / & 17 & $<0.0001$ \\
\hline \multicolumn{10}{|l|}{ Group 1} \\
\hline Age & 123 & 47.9 & \pm & 17.8 & 3 & 51.0 & \pm & 66.0 & 0.0357 \\
\hline $\operatorname{Sex}(F / M)$ & 123 & 99 & / & 24 & 3 & 2 & / & 1 & 0.1417 \\
\hline $\mathrm{mPAP}(\mathrm{mmHg})$ & 123 & 44.8 & \pm & 12.0 & 3 & 23.3 & \pm & 0.6 & $<0.0001$ \\
\hline PVR (WU) & 123 & 9.0 & \pm & 4.7 & 3 & 4.4 & \pm & 0.5 & $<0.0001$ \\
\hline PAWP $(m m H g)$ & 123 & 7.7 & \pm & 3.1 & 3 & 7.0 & \pm & 1.7 & 0.0359 \\
\hline $\mathrm{CO}(\mathrm{L} / \mathrm{min})$ & 123 & 4.7 & \pm & 1.5 & 3 & 3.8 & \pm & 0.7 & 0.1295 \\
\hline $6 \mathrm{MWD}(\mathrm{m})$ & 95 & 398.0 & \pm & 107.4 & 3 & 472.0 & \pm & 61.5 & 0.2394 \\
\hline VC,\% predicted & 111 & 85.6 & \pm & 16.9 & 3 & 91.4 & \pm & 9.5 & 0.8324 \\
\hline FEV1.0,\% predicted & 111 & 79.1 & \pm & 9.4 & 3 & 76.9 & \pm & 9.9 & 0.4183 \\
\hline DLCO/NA,\% predicted & 107 & 73.4 & \pm & 23.9 & 3 & 74.4 & \pm & 12.8 & 0.5192 \\
\hline $\mathrm{PaO}_{2}(\mathrm{mmHg})$ & 121 & 72.6 & \pm & 17.9 & 3 & 82.3 & \pm & 3.6 & 0.0013 \\
\hline $\mathrm{PaCO}_{2}(\mathrm{mmHg})$ & 121 & 36.6 & \pm & 5.3 & 3 & 39.3 & \pm & 0.7 & 0.0072 \\
\hline $\mathrm{P}_{\mathrm{V}} \mathrm{O}_{2}(\mathrm{mmHg})$ & 120 & 37.3 & \pm & 4.8 & 3 & 39.8 & \pm & 1.4 & 0.0003 \\
\hline $\mathrm{O}_{2}$ administration $(+)$ & & & $17(13.8 \%)$ & & & & $0(0 \%)$ & & 0.3479 \\
\hline $\mathrm{AaDo}_{2}(\mathrm{mmHg})$ & 121 & 33.9 & \pm & 19.2 & 3 & 21.0 & \pm & 4.4 & $<0.0001$ \\
\hline $\begin{array}{l}\text { WHO-FC } \\
(I / I / I I I / I V)\end{array}$ & 123 & & $(3 / 79 / 40 / 1)$ & & 3 & & $(0 / 3 / 0 / 0)$ & & 0.4546 \\
\hline Vasodilators $( \pm)$ & 123 & 92 & / & 31 & 3 & 2 & / & 1 & $<0.0001$ \\
\hline Underlying diseases & & & (\%) & & & & $(\%)$ & & \\
\hline IPAH/HPAH/PVOD/PCH & & 55 & 44.7 & & & - & - & & \\
\hline CTD & & 38 & 30.9 & & & 1 & 33.3 & & \\
\hline Congenital & & 16 & 13.0 & & & 1 & 33.3 & & \\
\hline Portal hypertension & & 12 & 9.8 & & & 0 & 0.0 & & \\
\hline drug/HIV & & 2 & 1.6 & & & 0 & 0.0 & & \\
\hline unknown & & & - & & & 1 & 33.3 & & \\
\hline \multicolumn{10}{|l|}{ Group 3} \\
\hline Age & 59 & 62.3 & \pm & 13.2 & 9 & 63.3 & \pm & 13.5 & 0.8404 \\
\hline $\operatorname{Sex}(F / M)$ & 59 & 28 & / & 31 & 9 & 5 & / & 4 & 0.6506 \\
\hline $\mathrm{mPAP}(\mathrm{mmHg})$ & 59 & 36.5 & \pm & 11.0 & 9 & 22.4 & \pm & 1.3 & 0.0003 \\
\hline PVR (WU) & 59 & 7.4 & \pm & 5.2 & 9 & 4.0 & \pm & 0.8 & 0.0546 \\
\hline $\operatorname{PAWP}(\mathrm{mmHg})$ & 59 & 4.5 & \pm & 3.2 & 9 & 5.7 & \pm & 3.7 & 0.12 \\
\hline
\end{tabular}


Table 6 (continued)

\begin{tabular}{|c|c|c|c|c|c|c|c|c|c|}
\hline \multirow{2}{*}{$\frac{\text { Total }}{\mathrm{CO}(\mathrm{L} / \mathrm{min})}$} & \multirow{2}{*}{$\frac{N}{59}$} & \multicolumn{3}{|c|}{ Conventional pre-capillry PH } & \multirow{2}{*}{$\begin{array}{l}\mathbf{n} \\
9\end{array}$} & \multicolumn{3}{|c|}{ Borderline pre-capillary PH } & \multirow{2}{*}{$\begin{array}{l}\text { p-value } \\
0.8225\end{array}$} \\
\hline & & 4.4 & \pm & 1.2 & & 4.3 & \pm & 1.2 & \\
\hline $6 \mathrm{MWD}(\mathrm{m})$ & 36 & 281.2 & \pm & 89.5 & 5 & 317.6 & \pm & 107.4 & 0.4098 \\
\hline VC,\% predicted & 52 & 59.8 & \pm & 24.2 & 7 & 66.9 & \pm & 33.5 & 0.4882 \\
\hline FEV1.0,\% predicted & 52 & 73.6 & \pm & 17.2 & 7 & 68.6 & \pm & 24.5 & 0.498 \\
\hline DLCO/NA,\% predicted & 43 & 42.0 & \pm & 30.0 & 6 & 51.9 & \pm & 18.7 & 0.4353 \\
\hline $\mathrm{PaO}_{2}(\mathrm{mmHg})$ & 53 & 64.4 & \pm & 35.4 & 9 & 65.8 & \pm & 15.6 & 0.9052 \\
\hline $\mathrm{PaCO}_{2}(\mathrm{mmHg})$ & 53 & 46.2 & \pm & 9.6 & 9 & 47.0 & \pm & 12.1 & 0.8248 \\
\hline $\mathrm{P}_{\mathrm{V}} \mathrm{O}_{2}(\mathrm{mmHg})$ & 53 & 34.9 & \pm & 4.0 & 9 & 35.1 & \pm & 3.0 & 0.9152 \\
\hline $\mathrm{O}_{2}$ administration $(+)$ & & & $17(2$ & & & & $1(1$ & & 0.2265 \\
\hline $\mathrm{AaDo}_{2}(\mathrm{mmHg})$ & 52 & 31.4 & \pm & 42.9 & 9 & 28.5 & \pm & 13.7 & 0.8458 \\
\hline $\begin{array}{l}\text { WHO-FC } \\
(\mathrm{I} / \mathrm{II} / \mathrm{II} / \mathrm{IV})\end{array}$ & & & $(0 / 1$ & & & & $(0 / 5$ & & 0.0671 \\
\hline Vasodilators $( \pm)$ & 59 & 35 & / & 24 & 9 & 0 & / & 9 & 0.0001 \\
\hline Underlying diseases & & & $(\%)$ & & & & (\%) & & \\
\hline ILD & & 36 & 61.0 & & & 4 & 44 & & \\
\hline COPD & & 13 & 22.0 & & & 3 & 33. & & \\
\hline $\mathrm{BE}$ & & 6 & 10.2 & & & 1 & 11. & & \\
\hline Others & & 4 & 6.8 & & & 1 & 11. & & \\
\hline \multicolumn{10}{|l|}{ Group 4} \\
\hline Age & 301 & 58.2 & \pm & 13.1 & 10 & 55.7 & \pm & 15.2 & 0.5475 \\
\hline $\operatorname{Sex}(F / M)$ & 301 & 216 & / & 85 & 10 & 8 & / & 2 & 0.5551 \\
\hline $\mathrm{mPAP}(\mathrm{mmHg})$ & 301 & 44.7 & \pm & 11.2 & 10 & 23.4 & \pm & 0.7 & $<0.0001$ \\
\hline PVR (WU) & 301 & 9.6 & \pm & 4.4 & 10 & 4.3 & \pm & 1.1 & 0.0002 \\
\hline PAWP(mmHg) & 301 & 7.5 & \pm & 3.1 & 10 & 5.3 & \pm & 2.4 & 0.0269 \\
\hline $\mathrm{CO}(\mathrm{L} / \mathrm{min})$ & 301 & 4.2 & \pm & 1 & 10 & 4.2 & \pm & 0.6 & 0.8073 \\
\hline $6 \mathrm{MWD}(\mathrm{m})$ & 254 & 357.8 & \pm & 99.4 & 4 & 458.5 & \pm & 80.4 & 0.0449 \\
\hline VC,\% predicted & 278 & 92.2 & \pm & 18.1 & 7 & 102.7 & \pm & 16 & 0.1311 \\
\hline FEV1.0,\% predicted & 278 & 75 & \pm & 9.3 & 7 & 78 & \pm & 10.2 & 0.4101 \\
\hline DLCO/NA,\% predicted & 267 & 82.3 & \pm & 24 & 7 & 85.2 & \pm & 15.6 & 0.7473 \\
\hline $\mathrm{PaO}_{2}(\mathrm{mmHg})$ & 296 & 60.7 & \pm & 18.2 & 10 & 80.1 & \pm & 11.1 & 0.0009 \\
\hline $\mathrm{PaCO}_{2}(\mathrm{mmHg})$ & 296 & 37.5 & \pm & 4.4 & 10 & 37 & \pm & 4.2 & 0.7296 \\
\hline $\mathrm{P}_{\mathrm{V}} \mathrm{O}_{2}(\mathrm{mmHg})$ & 296 & 33.1 & \pm & 1.2 & 10 & 38.2 & \pm & 3.9 & 0.0002 \\
\hline $\mathrm{O}_{2}$ administration $(+)$ & & & 34( & & & & $0(0$ & & 0.1248 \\
\hline $\mathrm{AaDo}_{2}(\mathrm{mmHg})$ & 296 & 44.8 & \pm & 19.1 & 10 & 25.9 & \pm & 8.1 & 0.002 \\
\hline $\begin{array}{l}\text { WHO-FC } \\
(\mathrm{I} / \mathrm{II} / \mathrm{II} / \mathrm{IV})\end{array}$ & & \multicolumn{3}{|c|}{$(3 / 138 / 153 / 7)$} & & & \multicolumn{2}{|c|}{$(0 / 9 / 1 / 0)$} & 0.0345 \\
\hline Vasodilators $( \pm)$ & 301 & 168 & / & 133 & 10 & 3 & / & 7 & 0.1049 \\
\hline PEA & 301 & 156 & / & 145 & 10 & 0 & / & 14 & 0.0002 \\
\hline BPA & 301 & 52 & / & 249 & 10 & 0 & / & 14 & 0.0536 \\
\hline Underlying diseases & & & $(\%)$ & & & & (\%) & & \\
\hline $\mathrm{PE}$ & & 279 & 92.7 & & & 9 & 90. & & \\
\hline Pulmonary stenosis & & 20 & 6.6 & & & 1 & 10. & & \\
\hline Others & & 2 & 0.7 & & & 0 & 0.0 & & \\
\hline
\end{tabular}

Data are expressed as mean $\pm S D$ or $n$ (\%). mPAP, mean pulmonary arterial pressure; PVR, pulmonary vascular resistance; PAWP, pulmonary arterial wedge pressure; $\mathrm{CO}$, cardiac output; 6MWD, 6-min walk distance; \%VC, percent vital capacity; FEV1.0\%, percent predicted forced expiratory volume in one second; \%DLCO/VA, diffusing capacity of carbon monoxide by the alveolar volume; $\mathrm{PaO}_{2}$, partial pressure of arterial oxygen; $\mathrm{PaCO}_{2}$, partial pressure of arterial oxygen; $\mathrm{PvO}_{2}$, mixed venous oxygen tension; $\mathrm{AaDO}_{2}$, alveolar-arterial oxygen difference; WHO-FC, World Health Organization Functional Class; IPAH, idiopathic pulmonary hypertension; $\mathrm{HPAH}$, hereditary pulmonary hypertension; PVOD, pulmonary veno-occlusive disease; $\mathrm{PCH}$, pulmonary capillary hemangiomatosis; CTD, connective tissue disease; ILD, interstitial lung disease; $C O P D$, chronic obstructive pulmonary disease; $\mathrm{BE}$, bronchiectasis; $\mathrm{PEA}$, pulmonary endarterectomy; PE, pulmonary embolism 

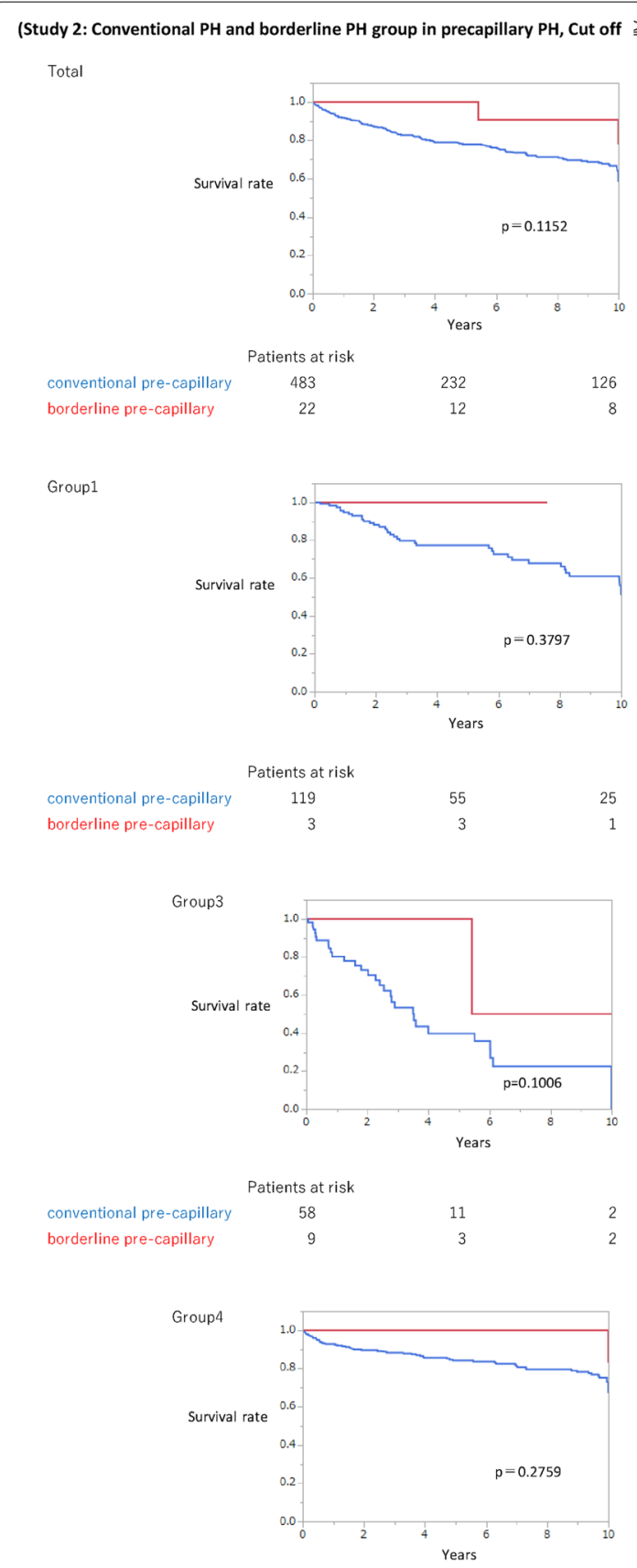

Patients at risk

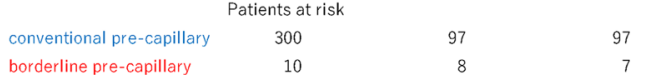

Fig. 3 Survival (Study 2: Conventional $\mathrm{PH}$ and borderline $\mathrm{PH}$ group in pre-capillary $\mathrm{PH}$, cut-off $\geq 3 \mathrm{WU}$ ). The survival of the conventional pre-capillary $\mathrm{PH}$ group was worse than that of the borderline pre-capillary PH group; however, the difference was not statistically significant. The same tendency was observed in Groups 1, 3 and 4. In Group 3, the 5-year and 10-year survival rates in the conventional pre-capillary PH population were $39.8 \%$ and $22.4 \%$, respectively. The survival rates in the borderline pre-capillary $\mathrm{PH}$ group were $100 \%$ and $50 \%$, respectively. $\mathrm{PH}$, pulmonary hypertension; WU, Wood units a higher percentage of borderline $\mathrm{PH}$ when the sample included more patients with cardiac diseases.

Assad et al. showed that among all patients, the percentage of those with mPAP between 19 and $24 \mathrm{mmHg}$, including non-PH patients $(20.1 \%)$ was $18 \%$, and that the majority of them belonged to Group 2 due to the presence of cardiovascular disease in $>70 \%$ of the patients [5]. Douschan et al. observed that $11.7 \%$ of all patients, including those without $\mathrm{PH}$ (35.2\%), had borderline $\mathrm{PH}$ [6]. In their study, $20.3 \%$ of patients belonged to Group 2 with overt $\mathrm{PH}$, and patients with borderline $\mathrm{PH}$ and those with overt $\mathrm{PH}$ showed a higher risk of cardiac disease. However, in these studies, detailed demographic data, including number of patients without $\mathrm{PH}$, were not known [5, 6]. Another study showed that the total percentage of patients with borderline $\mathrm{PH}$ was only $4.2 \%$, including non- $\mathrm{PH}$ patients (5.7\%) [7]. In that study, a relatively lower percentage of left heart disease (16.2\%) and a relatively higher percentage of respiratory disease (29.7\%) was observed among the patients with borderline $\mathrm{PH}$ and those without $\mathrm{PH}$. However, the background status of all patients, including those with overt $\mathrm{PH}$, is not known (Table 9) [7]. Only one study showed the proportion of patients with borderline pre-capillary $\mathrm{PH}$; however, the number was quite small and did not show the prognosis [9].

In our study, the number of patients with borderline $\mathrm{PH}$, including those in $\mathrm{PH}$ Groups $1-5$, was $8.6 \%$ lower than that reported in Assad's and Douschan's studies $(22.6 \%$ and $18.1 \%$, respectively) (Table 9$)$, and most of the total patients belonged to Group 3 (14.6\%) and 4 (55.6\%). The relatively higher ratio of these groups can be explained by our $\mathrm{PH}$ center being associated with respiratory medicine and being one of the high-volume PEA centers in Japan. Furthermore, the proportion of patients in Group 2, which is the most common form, was quite low. In our study, the ratio of borderline $\mathrm{PH}$ was relatively high in Group 3. Similarly, a study on 15 patients with severe COPD who underwent lung transplantation also showed a mild elevation of mPAP (20-25 $\mathrm{mmHg}$ ) in most patients [10]; therefore, the majority of patients with severe lung disease tended to have a mild elevation of mPAP. In contrast, our data showed that the ratio of borderline PH in Group 4 was low. The Papworth hospital study, which was also conducted in a PEA center, reported chronic thromboembolic disease with mPAP $<25 \mathrm{mmHg}$ in only 42 of 1019 patients (4.1\%) who underwent PEA [11]. The higher percentage of Group 4 patients in our center may explain the lower percentage of the borderline $\mathrm{PH}$ group in total. Overall, the number of patients who met the new diagnostic criteria depended on their background status. 
Table 7 Factors affecting the prognosis of Group 3 pre-capillary PH

\begin{tabular}{|c|c|c|c|c|}
\hline Factors & $\begin{array}{l}\text { Univariate crude hazard ratio } \\
(95 \% \mathrm{Cl})\end{array}$ & p-value & $\begin{array}{l}\text { Multivariate hazard ratio }(95 \% \\
\text { Cl) }\end{array}$ & $p$-value \\
\hline Age & $0.973(0.949-1.000)$ & 0.052 & $0.992(0.947-1.000)$ & 0.051 \\
\hline $\begin{array}{l}\text { Hemodynaics classification } \\
\text { (Conventional pre-capillary/Borderline } \\
\text { pre-capillary) }\end{array}$ & $4.690(0.623-35.284)$ & 0.055 & $4.265(0.547-33.238)$ & 0.09 \\
\hline $\begin{array}{l}\text { Time of diagnosis } \\
(\sim 2010 / 2010 \sim)\end{array}$ & $0.846(0.317-2.259)$ & 0.073 & $1.216(0.416-3.551)$ & 0.716 \\
\hline
\end{tabular}

PVR, pulmonary vascular resistance

Regarding baseline characteristics, in addition to hemodynamics, the $\mathrm{PaO}_{2}, \mathrm{PvO}_{2}$, and $\mathrm{AaDO}_{2}$ were better in the borderline pre-capillary $\mathrm{PH}$ group than in the conventional pre-capillary $\mathrm{PH}$ group. Lower $\mathrm{PaCO}_{2}$ in Group 1 of the conventional $\mathrm{PH}$ group might be suggestive of hyperventilation compensating for gas exchange impairment.

Several studies have shown little correlation between ventilatory function and severity of $\mathrm{PH}$ in patients with lung disease [12-14]. Similarly, in our study, there was no significant difference in ventilatory function between the conventional and the borderline pre-capillary $\mathrm{PH}$ groups in Group 3 (Table 6).

Regarding survival, in Study 1, the survival of the conventional PH group was worse than that of the borderline PH group. Similarly, in Study 2, the survival of the conventional pre-capillary $\mathrm{PH}$ group was worse than that of the borderline pre-capillary PH group; however, no significant difference was observed between the borderline $\mathrm{PH}$ and non- $\mathrm{PH}$ groups.

Previous data has suggested that mild elevation of $\mathrm{PH}$ is associated with poor prognosis in idiopathic pulmonary fibrosis [15] or chronic obstructive pulmonary disease $[16,17]$. Assad et al. also showed poor prognosis in patients with borderline $\mathrm{PH}$, and the majority of patients seemed to be in Group 2 [5]. Douchan et al. revealed poorer prognosis and increased cardiopulmonary comorbidities in patients with mPAP of $17-26 \mathrm{mmHg}$ than in those with $\mathrm{mPAP}<17 \mathrm{mmHg}$ [6]. They chose patients having a similar background status in both the $\mathrm{PH}$ and non-PH groups. Although a report including patients with relatively heterogeneous background diseases also showed poor prognosis of patients with borderline $\mathrm{PH}$, the difference in the prognosis between patients with overt $\mathrm{PH}$ and those with borderline $\mathrm{PH}$ was detected when they focused on patients with Group $1 \mathrm{PH}$ [7]. In our study, the patients in Group 3 had a poor prognosis, even in the non-PH group. Further, the number of nonPH patients was higher during 1999-2009 than that during 2010-2020, since we recently tended to perform RHC only in patients who may benefit from treatment with vasodilators. This means that most non- $\mathrm{PH}$ patients in 1999-2009 may not have received better treatment, compared with those diagnosed in 2010-2020. In addition, the number of patients who died due to malignant disease or who underwent lung transplantation was higher in the non-PH group. These underlying conditions may have affected the poor prognosis of the non-PH group in Group 3. Additionally, even when focusing on pre-capillary $\mathrm{PH}$ in Group 3, conventional $\mathrm{PH}$ vs. borderline $\mathrm{PH}$ and age were poor prognostic factors; however, the factors were not statistically significant. These data suggest that hemodynamics alone did not always determine the patients' prognosis in Group 3. Similarly, the Japanese Group $3 \mathrm{PH}$ registry revealed that in combined pulmonary fibrosis and emphysema, and interstitial pneumonia, the major cause of death was either respiratory failure or progression or acute exacerbation of underlying disease [18]. The higher percentage of patients in Group 3 in our study may also explain why there was no significant difference in survival between the conventional $\mathrm{PH}$ and non-PH groups (Fig. 2).

Concerning survival with different PVR cut-offs, Xanthouli et al. recently showed that patients with precapillary borderline PH with $P V R \geq 2$ WU had a significantly poorer prognosis than those with $\mathrm{PVR}<2 \mathrm{WU}$ in patients with systemic sclerosis [3]. Following this study, we compared the survival between patients with borderline pre-capillary $\mathrm{PH} \quad(25>\mathrm{mPAP}>20 \mathrm{mmHg}$ and PAWP $\leq 15 \mathrm{mmHg}$ ) with $\mathrm{PVR} \geq 3 \mathrm{WU}$ and those with PVR of 2-3 WU. There was no significant difference in survival between these two definition groups (Fig. 4). 
Fig. 4 Survival (Pre-capillary PH, PVR Cut-off: PVR $\geq 3$ WU vs. $3>P V R \geq 2 W U$ ). There was no significant difference between the patients with pre-capillary $\mathrm{PH}$ with $\mathrm{PVR} \geq 3 \mathrm{WU}$ and those with $3>P V R \geq 2$ WU. PH, pulmonary hypertension; WU, Wood units; PVR, pulmonary vascular resistance

Additionally, both patients died due to underlying diseases other than right heart failure. These data indicated that patients with PVR of 2-3 WU may not always have a good prognosis, which corresponds to the findings of Xanthouli et al. regarding patients with scleroderma. Further studies are needed to confirm whether this new definition is beneficial in clinical practice, including the cut-off for PVR.

Furthermore, the follow-up data for borderline $\mathrm{PH}$ and borderline pre-capillary $\mathrm{PH}$ was small, and very few patients were administered vasodilators during the follow-up (Table 8). The poor prognosis and unknown cause of death in these patients warrants the necessity for a closer follow-up to detect the progression of PH. Accordingly, prospective studies are needed to evaluate whether the new definition is valuable in identifying patients with $\mathrm{PH}$ and those who require prescription of vasodilators.

\section{Limitation}

This was a retrospective single-center study, and the sample size was relatively small to evaluate the pre-capillary PH group effectively. Furthermore, the possibility of selection bias could not be discounted, since our $\mathrm{PH}$ center specializes in respiratory medicine and the incidence of $\mathrm{PH}$ due to heat failure in this study was low. Further, we could not examine extensive lung disease by computed tomography in Group 3.

\section{Conclusions}

This is the first study performed in a $\mathrm{PH}$ center in an Asian country to reveal the characteristics of patients with pre-capillary $\mathrm{PH}$, according to the Nice 2018 definition. The Nice 2018 definition accounted for $4.3 \%$ of the patients with pre-capillary $\mathrm{PH}$, and most of them were in Groups 3 and 4. It was suggested that hemodynamics alone may not determine the patients' prognosis. Further prospective studies are needed to determine whether this new definition is beneficial in clinical practice and provides relevant information regarding prescription of $\mathrm{PH}$ specific treatment.
Total

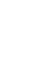

$P V R \geqq 3 W U$
$3>P V R \geqq 2$

Group1

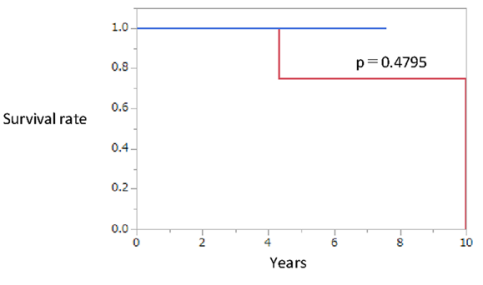

Patients at risk
$P V R \geqq 3 W U$

$3>P V R \geqq 2$

Group1 Collagen

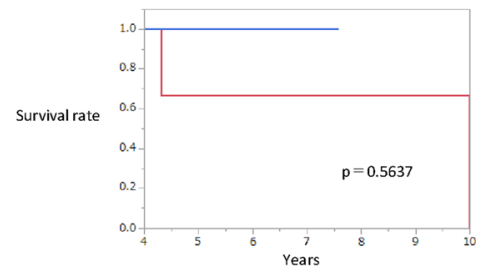

$P V R \geqq 3 W U$ 3> $P V R \geqq 2$
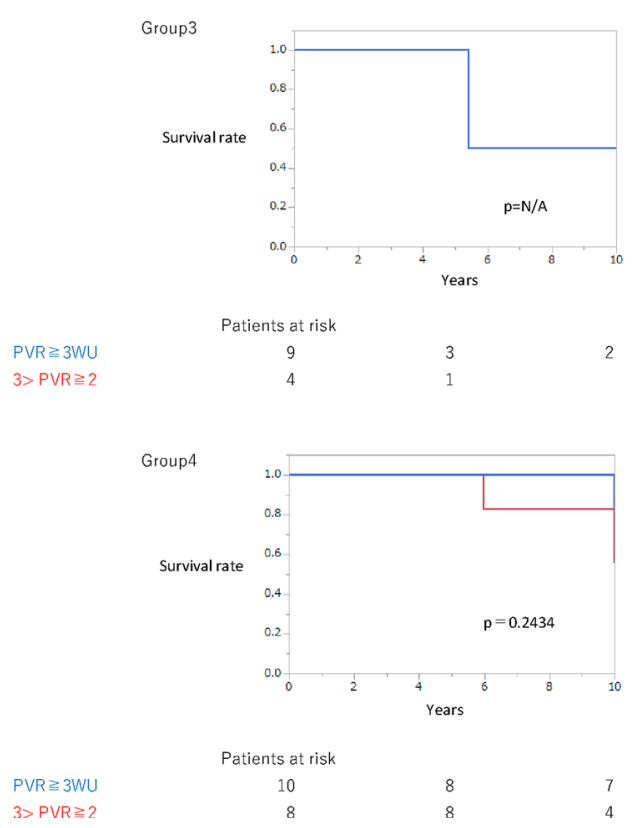


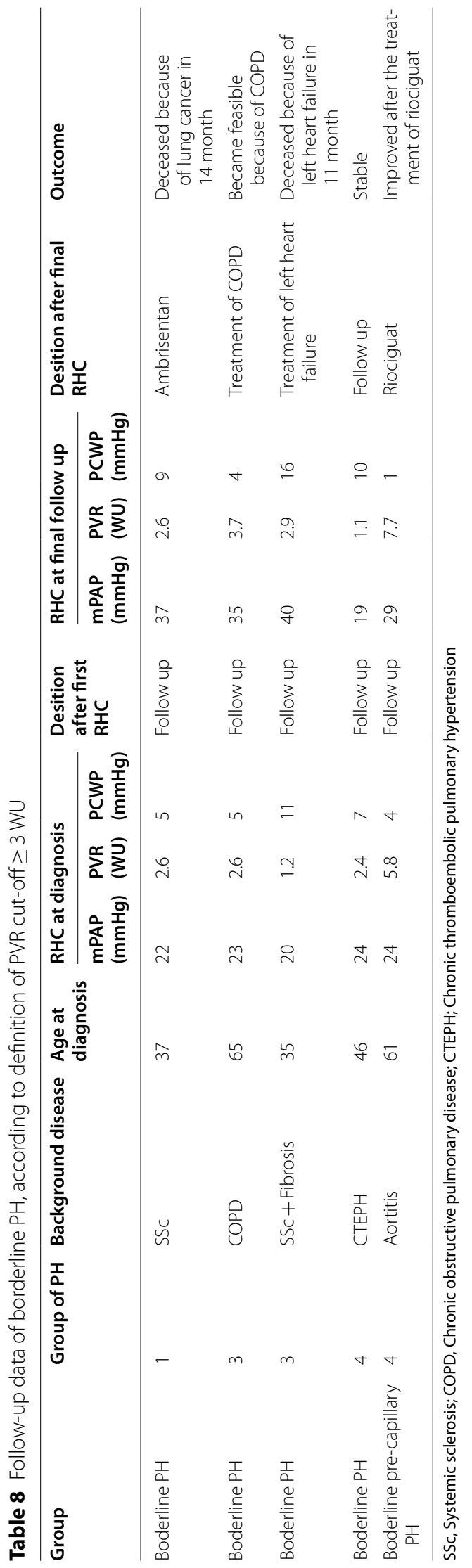


Table 9 Comparison with previous reports regarding the percentage of patients with "borderline pre-capillary PH" or "borderline $\mathrm{PH}$ "

\begin{tabular}{|c|c|c|c|c|c|c|c|}
\hline & Country & $\mathrm{n}$ & $\begin{array}{l}\% \text { of borde } \\
\text { capillary Pr }\end{array}$ & rline pre- & $\%$ of borderline $\mathrm{PH}$ & of total $\mathrm{PH}$ patients & Associated conditions \\
\hline & & & $\begin{array}{l}\text { of total } \\
\text { patients } \\
\text { (including } \\
\text { Non-PH) }\end{array}$ & $\begin{array}{l}\text { of total pre- } \\
\text { capillary } \mathrm{PH} \\
\text { patients }\end{array}$ & $\begin{array}{l}\text { of total patients } \\
\text { (including Non-PH) }\end{array}$ & & Total \\
\hline Assad et. al.(2017)[5] & U.S.A & 4343 & - & - & $18.0 \%$ & $22.6 \%$ & $\begin{array}{l}\text { CTD: } 0.9 \% \\
\text { CAD: } 71.5 \% \\
\text { COPD + ILD: } 18.7 \%\end{array}$ \\
\hline Douschan et.al.(2018)[6] & Austria & 547 & - & - & $11.7 \%$ & $18.1 \%$ & $\begin{array}{l}\text { ※Conventional group only } \\
\text { Group1:25.5\% } \\
\text { Group2:20.3\% } \\
\text { Group3:26.6\% } \\
\text { Group } 1: 18.3 \% \\
\text { Group5:9.3\% }\end{array}$ \\
\hline Gustavo et.al. (2013)[7] & the U.S & 1491 & - & - & $4.2 \%$ & $4.5 \%$ & $\begin{array}{l}\text { ※Borderline + borerline } \\
\text { precapillary only } \\
\text { None:30.4\% } \\
\text { CTD:16.2\% } \\
\text { Heart disease: } 16.2 \% \\
\text { Respiratory disease:29.7 }\end{array}$ \\
\hline Umit et.al. (2019)[9] & Turkey & 58 & $12.1 \%$ & $14.0 \%$ & Unknown & Unknown & $\begin{array}{l}\text { IPAH suspected: } 43.1 \% \\
\text { Congenital PH sus- } \\
\text { pected:34.5\% } \\
\text { Systemic sclerosis: } 3.4 \% \\
\text { Left heart disease and } \\
\text { valvular disease:6.9\% }\end{array}$ \\
\hline Our study & Japan & 683 & $3.2 \%$ & $4.3 \%$ & $7.3 \%$ & $8.6 \%$ & $\begin{array}{l}\text { Group1:22.8\% } \\
\text { Group2:3.1\% } \\
\text { Group3:14.6\% } \\
\text { Group4:55.6\% } \\
\text { Group5:1.2\% }\end{array}$ \\
\hline
\end{tabular}

CTD, connective tissue disease; CAD, coronary artery disease; COPD, chronic obstructive disease; ILD, interstitial lung disease; IPAH, idiopathic pulmonary hypertension; $\mathrm{PH}$, pulmonary hypertension

\section{Abbreviations}

WSPH: The World Symposium on Pulmonary Hypertension; PH: Pulmonary hypertension; mPAP: Mean pulmonary arterial pressure; RHC: Right heart catheterization; CO: Cardiac output; PAWP: Pulmonary arterial wedge pressure; PVR: Pulmonary vascular resistance; PEA: Pulmonary endarterectomy; ANOVA: One-way analysis of variance; $\mathrm{AaDO}_{2}$ : Alveolar-arterial oxygen difference; $\mathrm{PaCO}_{2}$ : Partial pressure of arterial carbon dioxide; $\mathrm{PaO}_{2}$ : Partial pressure of arterial oxygen; $\mathrm{PvO}_{2}$ : Partial pressure of mixed venous oxygen; WU: Wood unit.

\section{Acknowledgements}

This study was supported in part by grants from the Intractable Respiratory Diseases and Pulmonary Hypertension Research Group, the Ministry of Health, Labor and Welfare, Japan (H29-027), and the Pulmonary Hypertension Research Group of the Japan Agency for Medical Research and Development, AMED (17ek0019127h0003).

\section{Authors' contributions}

$K Y, N T$, and KT wrote the main manuscript text. KY and $Y T$ analyzed the data. NT, YT, AN, AS, RS, TJS, TS, AS, SS, and KT reviewed the manuscript. All authors read and approved the final manuscript.

\section{Funding}

Not applicable.

\section{Availability of data and materials}

The study database was anonymized, and the study complied with the requirements of the Japanese Ministry of Health, Labour and Welfare. The datasets analyzed during the current study are not publicly available, but are available from the corresponding author on a reasonable request and with the permission of our department.

\section{Declarations}

\section{Ethics approval and consent to participate}

We protected the identity of patients' compiled data according to the requirements of the Japanese Ministry of Health, Labor, and Welfare, dedicated to privacy, information technology, and civil rights. The Research Ethics Committee of Chiba University School of Medicine approved the study's protocol (approval number 2,584). Since 2009, all survivors provided written informed consent for a prospective cohort study (approval number 826 ). Deaths before 2008 were not required to provide written informed consent as per the guidelines for retrospective studies in Japan and the ethics committee of Chiba University Hospital permit it (Study 2). The study database was anonymized. All experiments were performed in accordance with the relevant guidelines and regulations.

\section{Consent for publication}

Not applicable.

\section{Competing interests}

The authors declare that they have no competing interests.

\section{Author details}

${ }^{1}$ Department of Respirology, Graduate School of Medicine, Chiba University, 1-8-1 Inohana Chuou-ku, Chiba 260-8670, Japan. ${ }^{2}$ Groupe de Recherche en Hypertension Artérielle Pulmonaire, Institut Universitaire de Cardiologie Et 
de Pneumologie de Québec - Université Laval, Quebec, Canada. ${ }^{3}$ Saiseikai Narashino Hospital, Narashino-shi, Izumi-cho, Chiba 275-8580, Japan. ${ }^{4}$ Vrije Universiteit Medische Centrum, De Boelelaan 1117, 1118, 1081 HV Amsterdam, The Netherlands.

Received: 4 March 2021 Accepted: 26 July 2021

Published online: 09 August 2021

\section{References}

1. Simonneau G, Montani D, Celermajer DS, Denton CP, Gatzoulis MA, Krowka M, et al. Haemodynamic definitions and updated clinical classification of pulmonary hypertension. Eur Respir J. 2019;53.

2. Kovacs G, Berghold A, Scheidl S, Olschewski H. Pulmonary arterial pressure during rest and exercise in healthy subjects: a systematic review. Eur Respir J. 2009;34:888-94.

3. Xanthouli P, Jordan S, Milde N, Marra A, Blank N, Egenlauf B, et al. Haemodynamic phenotypes and survival in patients with systemic sclerosis: the impact of the new definition of pulmonary arterial hypertension. Ann Rheum Dis. 2020;79:370-8.

4. Jenkins D, Madani M, Fadel E, D'Armini AM, Mayer E. Pulmonary endarterectomy in the management of chronic thromboembolic pulmonary hypertension. Eur Respir Rev. 2017:26.

5. Assad TR, Maron BA, Robbins IM, Xu M, Huang S, Harrell FE, et al. Prognostic effect and longitudinal hemodynamic assessment of borderline pulmonary hypertension. JAMA Cardiol. 2017;2:1361-8.

6. Douschan P, Kovacs G, Avian A, Foris V, Gruber F, Olschewski A, et al. Mild elevation of pulmonary arterial pressure as a predictor of mortality. Am J Respir Crit Care Med. 2018;197:509-16.

7. Heresi GA, Minai OA, Tonelli AR, Hammel JP, Farha S, Parambil JG, et al. Clinical characterization and survival of patients with borderline elevation in pulmonary artery pressure. Pulm Circ. 2013;3:916-25.

8. Wijeratne DT, Lajkosz K, Brogly SB, Lougheed MD, Jiang L, Housin A, et al. Increasing incidence and prevalence of world health organization groups 1 to 4 pulmonary hypertension: a population-based cohort study in Ontario, Canada. Circ Cardiovasc Qual Outcomes. 2018;11:e003973.

9. Sinan ÜY, Çetinarslan Ö, Arat Özkan A, Ersanlı MK, Küçükoğlu MS. The impact of the new World Symposium on Pulmonary Hypertension definition of pulmonary hypertension on the prevalence of pre-capillary pulmonary hypertension. Turk Kardiyoloji Dernegi arsivi: Turk Kardiyoloji Derneginin yayin organidir. 2019;47:594-8.

10. Andersen KH, Iversen M, Kjaergaard J, Mortensen J, Nielsen-Kudsk JE,

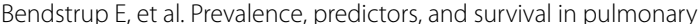
hypertension related to end-stage chronic obstructive pulmonary disease. J Heart Lung Transplant. 2012;31:373-80.

11. Taboada D, Pepke-Zaba J, Jenkins DP, Berman M, Treacy CM, Cannon JE, et al. Outcome of pulmonary endarterectomy in symptomatic chronic thromboembolic disease. Eur Respir J. 2014;44:1635-45.

12. Nathan SD, Shlobin OA, Ahmad S, Urbanek S, Barnett SD. Pulmonary hypertension and pulmonary function testing in idiopathic pulmonary fibrosis. Chest. 2007:131:657-63.

13. Low AT, Medford AR, Millar AB, Tulloh RM. Lung function in pulmonary hypertension. Respir Med. 2015;109:1244-9.

14. Hurdman J, Condliffe R, Elliot CA, Swift A, Rajaram S, Davies C, et al. Pulmonary hypertension in COPD: results from the ASPIRE registry. Eur Respir J. 2013;41:1292-301.

15. Hamada K, Nagai S, Tanaka S, Handa T, Shigematsu M, Nagao T, et al. Significance of pulmonary arterial pressure and diffusion capacity of the lung as prognosticator in patients with idiopathic pulmonary fibrosis. Chest. 2007;131:650-6.

16. Kessler R, Faller M, Fourgaut G, Mennecier B, Weitzenblum E. Predictive factors of hospitalization for acute exacerbation in a series of 64 patients with chronic obstructive pulmonary disease. Am J Respir Crit Care Med. 1999;159:158-64.

17. Oswald-Mammosser M, Weitzenblum E, Quoix E, Moser G, Chaouat A, Charpentier $C$, et al. Prognostic factors in COPD patients receiving longterm oxygen therapy. Importance of pulmonary artery pressure. Chest. 1995;107:1193-8.

18. Tanabe N, Kumamaru H, Tamura Y, Taniguchi H, Emoto N, Yamada Y, et al. Multi-institutional prospective cohort study of patients with pulmonary hypertension associated with respiratory diseases. Circ J. 2021;85:333-42.

\section{Publisher's Note}

Springer Nature remains neutral with regard to jurisdictional claims in published maps and institutional affiliations.
Ready to submit your research? Choose BMC and benefit from:

- fast, convenient online submission

- thorough peer review by experienced researchers in your field

- rapid publication on acceptance

- support for research data, including large and complex data types

- gold Open Access which fosters wider collaboration and increased citations

- maximum visibility for your research: over 100M website views per year

At $B M C$, research is always in progress.

Learn more biomedcentral.com/submissions 